\title{
On the Structural Analysis of $\boldsymbol{\gamma}$-Ray Induced Primary Free Radicals in UHMWPE and Vitamin E Stabilized UHMWPE by ESR Spectroscopy
}

\author{
Malik Sajjad Mehmood, ${ }^{1,2,3}$ Muhammad Shah Jahan, ${ }^{2}$ Tariq Yasin, ${ }^{3}$ Muhammad Tariq, \\ Mohammad Ahmad Choudhry, ${ }^{1}$ and Masroor Ikram ${ }^{3}$ \\ ${ }^{1}$ University of Engineering and Technology, Taxila 47050, Pakistan \\ ${ }^{2}$ Biomaterials Research Laboratory, University of Memphis, Memphis, TN 38152, USA \\ ${ }^{3}$ Pakistan Institute of Engineering and Applied Science, Islamabad 45650, Pakistan
}

Correspondence should be addressed to Malik Sajjad Mehmood; msajjad.82@gmail.com

Received 26 October 2014; Revised 22 February 2015; Accepted 1 March 2015

Academic Editor: E. Cristina Stanca-Kaposta

Copyright (C) 2015 Malik Sajjad Mehmood et al. This is an open access article distributed under the Creative Commons Attribution License, which permits unrestricted use, distribution, and reproduction in any medium, provided the original work is properly cited.

\begin{abstract}
Oriented allyl radicals are detected at room temperature in gamma irradiated UHMWPE. The effects of vitamin E and storage at room temperature on this oriented structure are also investigated during the study. While testing powder as well as compressionmolded solids, with or without vitamin E, a typical ESR spectrum was recorded at room temperature following $100 \mathrm{kGy}$ gamma dose and subsequent storage at $-78.5^{\circ} \mathrm{C}$ for one year. The simulated results show that the relative abundance of $5 \%$ alkyl, $68 \%$ allyl, and $27 \%$ polyenyl produced a $98.7 \%$ best fit of experimental ones. Furthermore, the allyl radical signal gives approximately $20 \%$ of random orientations and $80 \%$ of oriented molecules. In oriented PE, measured at $-196^{\circ} \mathrm{C}$, Ohnishi et al. (1916) observed 25 lines within a total magnetic field width of approximately $133 \mathrm{G}$. Our spectra also show 25 lines spread over $136 \mathrm{G}$ in UHMWPE powder samples and at room temperature after one year of storage.
\end{abstract}

\section{Introduction}

In total joint replacement devices, adhesive/abrasive wear of ultra-high molecular weight polyethylene (UHMWPE) components is the major problem associated with their long term performance. To increase the wear resistance of these components, cross-linking using ionization radiation has been used and clinically available UHMWPE is cross-linked at radiation doses ranging from $50 \mathrm{kGy}$ to $100 \mathrm{kGy}$. The crosslinking of polyethylene (PE) is achieved by the recombination of radiation induced free radicals in the amorphous phase; however, some free radicals become trapped in the crystalline core of UHMWPE for the prolonged period of time [1]. These free radicals (along with cross-linking/recombination during their migration process from crystalline core to amorphous phase) are involved in other complicated secondary reactions, thus resulting in radiation chemical effects such as main chain degradation by oxidation, gas evolution, unsaturation in chemical bonding, and so forth. Although there are some established methods to study the secondary products generated as a result of free radical reactions but to have clear insight on the radiation chemistry of UHMWPE, it is very important to study primary products of radiation treatment, that is, free radicals themselves. In this regard, radical analysis technique, known as electron paramagnetic resonance (EPR) or electron spin resonance (ESR), is the only and most suitable technique for the direct detection of free radicals [2-4].

In this study, the primary trapped free radical inside the medical grade UHMWPE as a result of radiation crosslinking has been studied in detail while using ESR spectroscopy. The ESR spectra have been analyzed in detail with testing the irradiated samples at extremely low microwave 
powers and computer simulation. The materials which are used in this study were virgin UHWMPE powder and compression molded block of UHMWPE. In addition, the effects of vitamin $\mathrm{E}$ and storage at room temperature on the primary radical's structures have been investigated. The behavior of spectra as a function of storage time has been monitored for concluding the effect of storage temperature and presence of vitamin $\mathrm{E}$ on these primary radicals.

\section{Materials and Methods}

2.1. Material, Irradiation, and Storage. Pristine UHMWPE powder (GUR 1020), vitamin E ( $0.1 \%$ by wt.) blended UHMWPE powder (GUR 1020E), and pristine solid UHMWPE sample (prepared from compression moulded bar stock made of GUR 1020) were used during this study. All samples were provided by Ticona in powder form and used as such without further treatment to investigate the radical chemistry of UHMWPE. All samples were irradiated with gamma rays using ${ }^{60} \mathrm{Co}$ source with a total dose of either $65 \mathrm{kGy}$ or $100-\mathrm{kGy}$. The samples were enclosed in gas impermeable nitrogen filled Mylar foil bags before sending to irradiation department and remained in these nitrogen-filled Mylar foil bags during irradiation.

Irradiation services were provided by the Steris-Isomedix Services (Grove Park, OH, USA), and, immediately after receiving from irradiation department, the samples were stored either at $-78.5^{\circ} \mathrm{C}$ (dry ice temperature) or at $-196^{\circ} \mathrm{C}$ (liquid nitrogen temperature) for approximately one year to freeze the radical's mobility to some extent. It is worth to mention here that majority of $\mathrm{PE}$ radicals does decay via recombination with each other; however; decay by oxidation reactions is not possible because of their storage in inert atmosphere during and after irradiation.

2.2. Experimental Measurements. For free radical measurements the samples were taken out from the cold (dry ice/liquid nitrogen) environment and brought to the room temperature. The powder fills approximately $10.0 \mathrm{~mm}$ of ESR sample tube with internal diameter of $3.8 \mathrm{~mm}$ and weight $4.33 \mathrm{mg}$. The weight of the powder samples was approximately 65-68 mg. The size and weight of solid samples used for ESR measurements were $2 \times 2 \times 8 \mathrm{~mm}^{3}$ and $40 \mathrm{mg}$, respectively. All this procedure was performed inside a large nitrogenfilled/inflated glove bag to minimize the samples exposure to oxygen at first measurement.

Free radicals were detected at room temperature using a highly sensitive, X-band ESR spectrometer (EMX 300 by Bruker). The spectrometer was fitted with a high-sensitive, mixed mode cavity and operates at $9.8 \mathrm{GHz}$ microwave frequency and $100 \mathrm{kHz}$ modulation and detection frequencies. All ESR spectra (first derivative of absorption) were recorded at $0.01-1 \mathrm{~mW}$ microwave power and at $2 \mathrm{G}$ modulation amplitude.

After the initial measurements, samples were stored in open air at room temperature and radical decay via oxidation reaction in the presence and absence of vitamin $\mathrm{E}$ was monitored with time up to eight weeks.

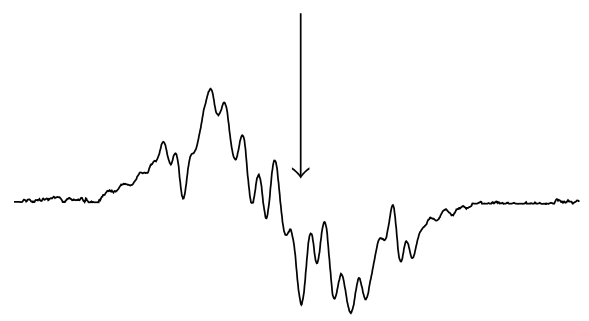

(a)

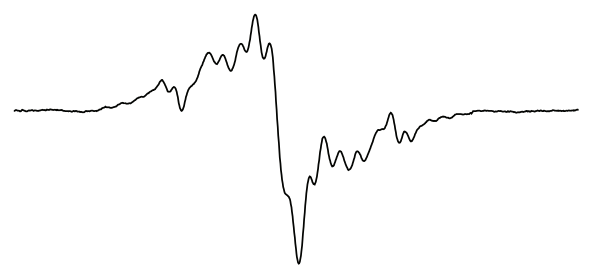

(b)

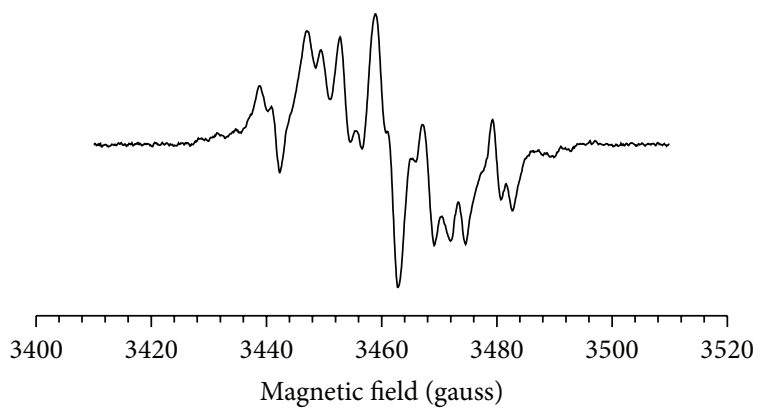

(c)

FIGURE 1: ESR spectra of $100 \mathrm{kGy}$ irradiated samples, (a) pristine UHMWPE powder samples, (b) vitamin E UHMWPE samples, and (c) compression moulded UHMWPE sample.

\section{Results and Discussions}

Figure 1 shows the ESR spectra of the $100 \mathrm{kGy}$ gamma irradiated pristine and vitamin E blended UHMWPE powder along with compression moulded pristine UHMWPE sample. All samples were stored at either at $-78.5^{\circ} \mathrm{C}$ (UHMWPE powder samples) or at $-196^{\circ} \mathrm{C}$ (UHMWPE solid samples) after irradiation. The irradiation was performed at room temperature with an average dose rate of $7.5 \mathrm{kGy} / \mathrm{hr}$ and measurements were also performed at room temperature. The samples were brought to room temperature before testing as mentioned in Section 2.2. The observed ESR spectra consist of additional hyperfine lines (super hyperfine (shf) lines) superimposed on the primary PE radical signals. In addition to this, the central single line due to polyenyle radical [5] is absent as indicated by arrow in Figure 1. These shf lines are also there in vitamin E containing samples and vitamin E radicals signal are found on the top of these shf lines of PE radicals (see Figure 1(b)). A similar ESR pattern is observed in $100 \mathrm{kGy}$ irradiated compression molded sample (see Figure 1(c)); however, the only difference in compression moulded UHMWPE is that this shf line ESR pattern disappears at slower rate as compared to 


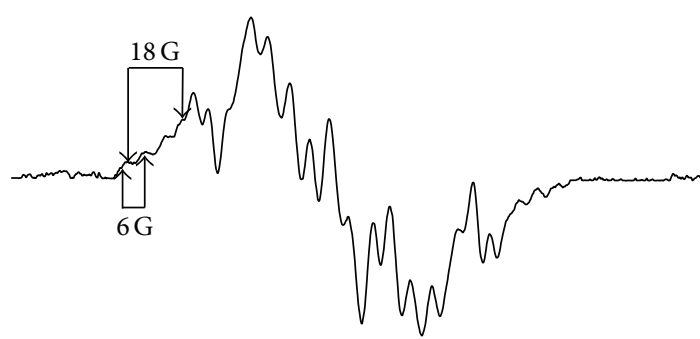

FIGURE 2: ESR spectra of $100 \mathrm{kGy}$ pristine UHMWPE powder.

pristine and vitamin E blended powder UHMWPE. The oxygen penetration rate from air to UHMWPE which is higher in powder UHMWPE samples as compared to compression moulded is mainly responsible and can be used to explain and understand this particular difference in both groups, that is, compression moulded solid and powder samples. The appearance of shf lines at the top of PE radical's signals in these after long postirradiation storage, that is, approximately one year, is quite interesting as far as role of radiation induced free radicals in PE modification is concerned. In order to analyze these complex ESR spectra, ESR power saturation technique along with computer aided simulation has been employed. The advantage of ESR power saturation technique is that using this technique radical's species can be isolated for qualitative and quantitative analysis [4-6].

The first ESR test has been performed on the $100 \mathrm{kGy}$ pristine UHMWPE powder sample and this test is performed with extreme care to avoid the sample exposure with oxygen or air. The data was taken under common ESR setting, that is, at $1 \mathrm{~mW}$ microwave power and $2 \mathrm{G}$ modulation amplitude. ESR spectrum for this initial test is quite complex with addition lines so-called shf lines superimposed on the main septet spectrum of already reported allyl radical (see Figure 2 for detail). The appearance of this complex ESR spectrum after one year of storage enhances the curiosity due to the reason that under these common ESR setting (i.e., at $1 \mathrm{~mW}$ microwave power and $2 \mathrm{G}$ modulation amplitude), $\mathrm{PE}$ spectrum is septet which is reported so far as the combination of seven-line allyl radical and singlet of polyenyle radical [7]. A similar kind of situation has been found for vitamin $\mathrm{E}$ bended UHMWPE powder sample which is also of major concern because it suggests that even vitamin $\mathrm{E}$ has no effect on radical behavior during the storage time. All these observations after initial tests on powder UHMWPE samples suggest that these radicals were trapped inside the crystalline core where the role of vitamin $\mathrm{E}$ is reported to be limited as free radical quencher [1]. The in depth analysis of left most side the ESR spectrum (because either left most or right most area/side remains unaffected from overlapping signals of different species) shows that main lines with $18 \mathrm{G}$ separations have further doublet structure with a separation of $6 \mathrm{G}$.

However, on testing the compression moulded solid pristine sample under the same common ESR setting as mentioned above this complex ESR spectrum is missing. The plausible explanation for this is that at normal microwave power setting to monitor the $\mathrm{PE}$ radicals is $1 \mathrm{~mW}$. At this value the signals of different radicals overlap [6] and further splitting is either suppressed by the other species or by power saturation effect. In order to further investigate and for our satisfaction regarding aforementioned facts, testing of solid compression moulded samples has been performed at various low microwave power settings. The additional lines start appearing on going below $1 \mathrm{~mW}$, and at $0.01 \mathrm{~mW}$ ESR spectrum with shf lines can be seen with minimum influence of signal suppression due to overlapping and/or microwave power saturation (see Figure 3).

It can be seen in Figure 3 and zoomed in portion of Figure 4 that the spectrum of this particular sample at $0.01 \mathrm{~mW}$ has seven lines as main components. The separation between main lines was found to be approximately $18 \mathrm{G}$ and each peak of this spectrum has further doublet splitting of approximately $6 \mathrm{G}$ as can be seen in the enlarged portion of the right most side of ESR spectrum (see Figure 4) because it was reported that the splitting of outermost peaks as well as the splitting of outermost doublet remained constant [7]. These initial tests conclude that the appearance of further doublet splitting or shf lines is independent of samples morphology and appears for both samples weather it is powder or compression moulded solid one; however, it does depend on absorbed dose because, for $65 \mathrm{kGy}$ irradiated UHMWPE samples (powder and solid, pristine and vitamin E blended), this shf line structure is missing as shown in Figure 5.

For the analysis of shf lines or further splitting of main polyethylene line into doublet, the outmost portion of $100 \mathrm{kGy}$ spectra (weather powder or solid) has been focused through the testing of samples. This has been done by zooming into the areas of interest of each spectrum and found that the separation of the main line on the magnetic field axis is approximately $18 \mathrm{G}$ which is further divided into the double lines having the separation of approximately $6 \mathrm{G}$. This supports that this spectrum is due to allyl radical with complex doublet splitting of septet due to $\alpha_{1}$ proton hf coupling. The allyl radical is distributed over three carbons atom as shown in Figure 6. It interacts with three hydrogen atoms at $\alpha$ position (one at $\alpha_{1}$ and two at $\alpha_{2}$ position) and four $\beta$ hydrogen (two $\beta_{1}$ and two $\beta_{2}$ ) with different hf constants $[7,8]$. The hyperfine values for six hydrogen atoms, that is, two at $\alpha$ position and four at $\beta$ position, give the seven-line ESR spectrum and the contribution from $\alpha_{1}$ hydrogen atom results in doublet structure.

For theoretical confirmations of the abovementioned spectrum analysis and appearance of shf lines due to $\alpha_{1}$ proton in allyl radicals, spectrum deconvolution has been performed using WinSim software [9]. The best simulated spectra along with the fitting parameters for pristine and vitamin E blended UHMWPE are shown in Figure 7 and Table 1, respectively. The fitting involves the PE radicals including allyl, polyenyle, and vitamin $\mathrm{E}$ radicals. The best fit (with fitting correlation of 0.94) of the experimental spectra using WinSim program [9] gives the hf constants of $\alpha_{1}$, $\alpha_{2}, \beta_{1}$, and $\beta_{2}$ for allyl radicals as $5.9,17.2,23.6$, and 12.7, respectively. These values of $\mathrm{hf}$ constants for allyl radical are very close to the already reported one for allyl radicals in 


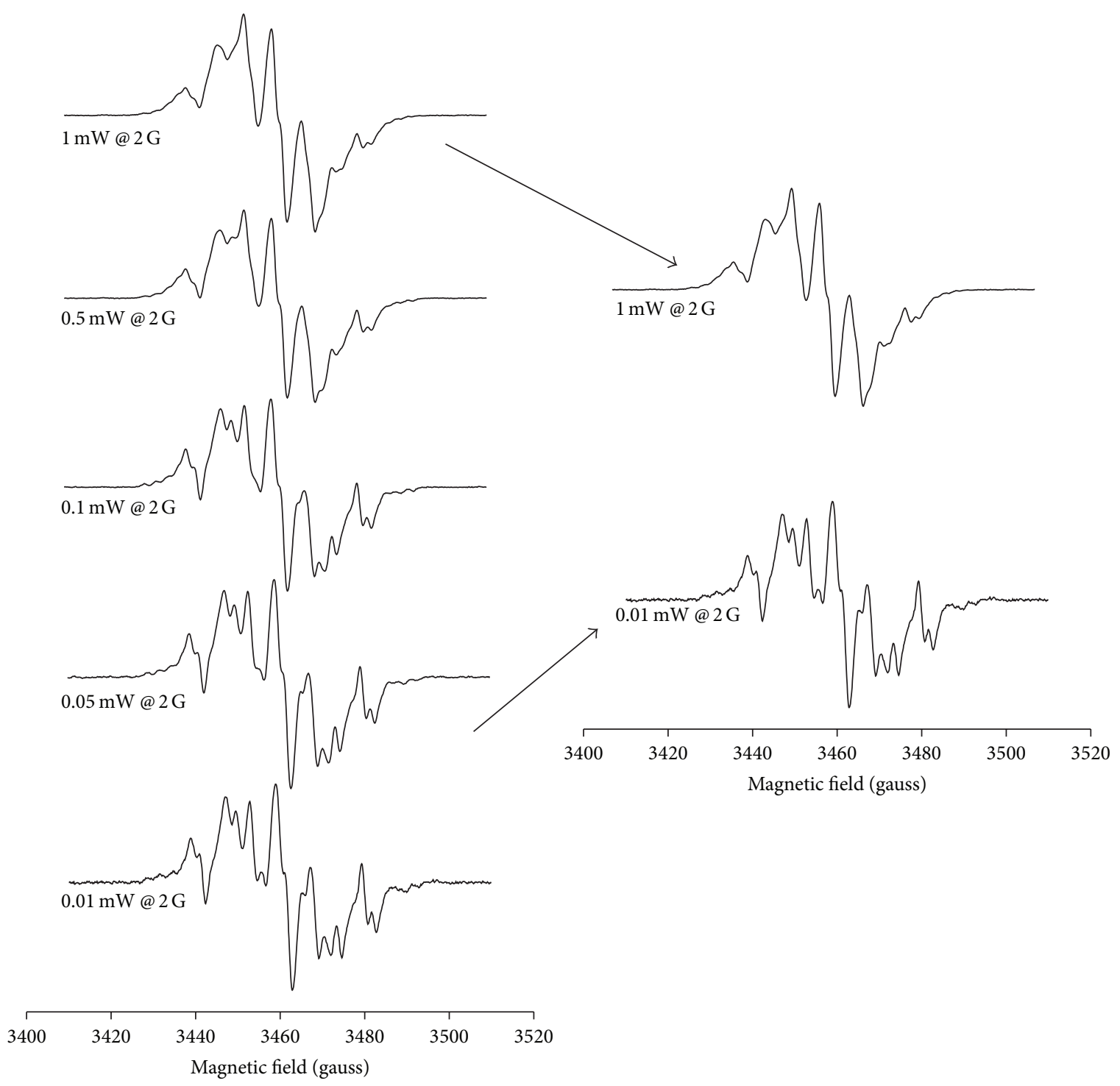

FIGURE 3: ESR microwave power saturation behavior of super hyperfine pattern ESR pattern.

highly oriented polyethylene fibers at $+11^{\circ} \mathrm{C}$, that is, $5.51,21.3$, 24.4 , and 17.4 [7]. It is worth to mention here that these tests were performed at room temperature (i.e., approximately at $\left.+25^{\circ} \mathrm{C}\right)$.

The reported results of allyl radical hf coupling constants here in this study are quite different from recently reported results of Zhao et al. [8]. They have observed 11 for allyl radicals because of small hf coupling constant of $\alpha_{1}$ proton in highly oriented UHMWPE fiber. However, this study reveals that allyl radical is distributed over three carbons (see Figure 6), interacting with three hydrogen atoms at $\alpha$ position (one at $\alpha_{1}$ and two at $\alpha_{2}$ position) and four $\beta$ hydrogen (two $\beta_{1}$ and two $\beta_{2}$ ) with different hf constants $[7,8]$. The hyperfine values for six hydrogen atoms, that is, two at $\alpha$ position and four at $\beta$ position, give the seven line ESR spectrum and the contribution from $\alpha_{1}$ hydrogen atom results in doublet structure. These observations are for unoriented UHMWPE pristine and vitamin E powder samples and compression moulded sample and only for $100 \mathrm{kGy}$ irradiated samples. Moreover, this complex spectrum of allyl radical has been observed after one year of storage and at room temperature, that is, approximately at $25^{\circ} \mathrm{C}$. On the basis of these observations along with the reported ESR spectra of $\mathrm{PE}$ allyl radicals in the literature for highly oriented UHMWPE/PE fiber samples, one can conclude that irradiating the unoriented even powder samples of UHMWPE with a dose of $100 \mathrm{kGy}$ can induce orientation of crystalline lamellae to some extent which is stable at room temperature.

In order to monitor the stability of this oriented structure $100 \mathrm{kGy}$ irradiated with storage time, the behavior of shf ESR pattern and the ESR spectra of $65 \mathrm{kGy}$ and $100 \mathrm{kGy}$ irradiated samples has been monitored for eight weeks (see Figure 8).

Free radical decay in the samples with $100 \mathrm{kGy}$ of absorbed dose follows the same trend as that of $65 \mathrm{kGy}$ except for the first week of storage. The shf patterns in pristine UHMWPE samples disappear in 24 hours and septet spectra 
TABLE 1: Relative concentration of radicals, spin 1/2 coupling constants, \% lorentzian, and line width for 100 kGy irradiated pristine UHMWPE and vitamin E blended UHMWPE powder used as input for WinSim simulation software.

\begin{tabular}{|c|c|c|c|c|c|c|}
\hline \multirow{2}{*}{ Sample/radical } & \multicolumn{3}{|c|}{ Pristine UHMWPE } & \multicolumn{3}{|c|}{ Vitamin E blended UHMWPE } \\
\hline & Alkyl & Allyl & Polyenyle & Allyl & Polyenyle & Vitamin $\mathrm{E}$ \\
\hline Rel. Conc. & $5.30 \%$ & $68.10 \%$ & $26.60 \%$ & $43.20 \%$ & $42.40 \%$ & $14.40 \%$ \\
\hline $\mathrm{H}_{\alpha 1}$ & $1 @ 23.1 \mathrm{G}$ & $1 @ 5.9 \mathrm{G}$ & - & $1 @ 5.7 \mathrm{G}$ & - & - \\
\hline $\mathrm{H}_{\alpha 2}$ & $4 @ 31.7 \mathrm{G}$ & $2 @ 17.2 \mathrm{G}$ & - & $2 @ 18 \mathrm{G}$ & - & $3 @ 4.9 \mathrm{G}$ \\
\hline $\mathrm{H}_{\beta 1}$ & - & $2 @ 23.6 \mathrm{G}$ & - & $2 @ 26 \mathrm{G}$ & - & - \\
\hline $\mathrm{H}_{\beta 2}$ & - & $2 @ 12.7 \mathrm{G}$ & - & $2 @ 15 \mathrm{G}$ & - & $3 @ 6.3 \mathrm{G}$ \\
\hline G-shift & -3.70 & -3.15 & -10.5 & -5.5 & -11.5 & -7.03 \\
\hline$\%$ lorentzian & 0 & 93 & 7 & 100 & 59 & 0 \\
\hline Line width $(\mathrm{G})$ & 5.26 & 4.3 & 18 & 4.3 & 19.5 & 2.5 \\
\hline
\end{tabular}

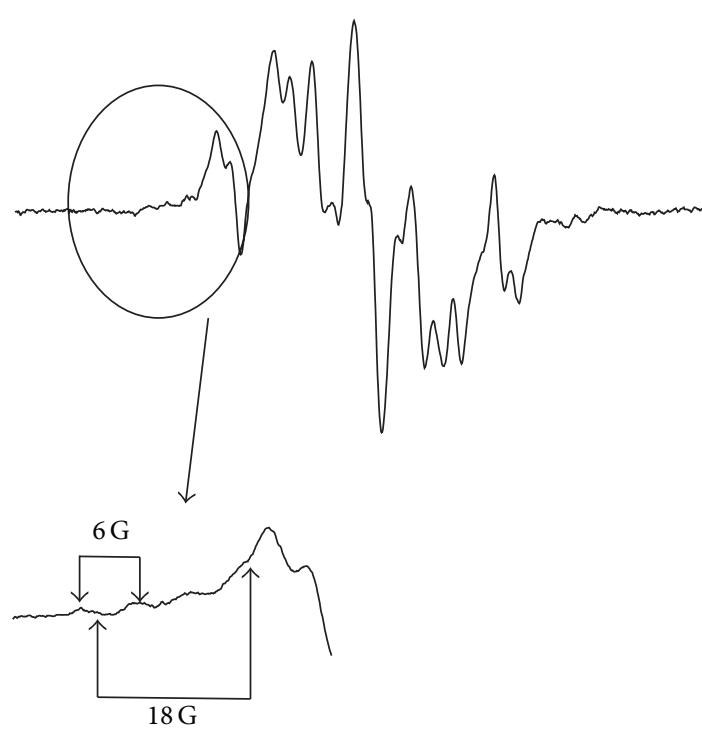

FIGURE 4: (a) ESR spectra of 100-kGy irradiated sample at $0.01 \mathrm{~mW}$ microwave power. (b) The right most portion of the spectrum is shown with line separations.

without further complex splitting appear which turns into seven lines in about one week of storage. The initial trend of shf pattern is similar in vitamin E containing sample; however, the observed spectrum after 24 hours for vitamin $\mathrm{E}$ containing sample is quite different from virgin. The reaction of peroxy radical (formed during the oxidative decay of allyl radical) with vitamin $\mathrm{E}$ may be the reason for this difference [10-14]. In addition to investigating the effect of radiation dose and storage time initial measurements of thermal annealing in the presence of vitamin $\mathrm{E}$ were also performed. As thermal annealing enhances the main UHMWPE chain mobility and accelerates the decay rate of free radicals, the addition of vitamin E plays the supportive role for the mobility of chains due to its plasticizing nature. Therefore, rate of radical decay due to thermal annealing is higher in vitamin E doped UHMWPE as compared to pristine one. Moreover, vitamin E in excess amount has been found to decrease the free radical concentration but its higher concentration is responsible for reduction in $\mathrm{PE}$

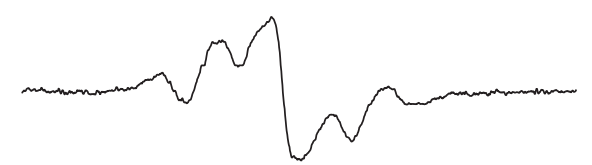

(a)
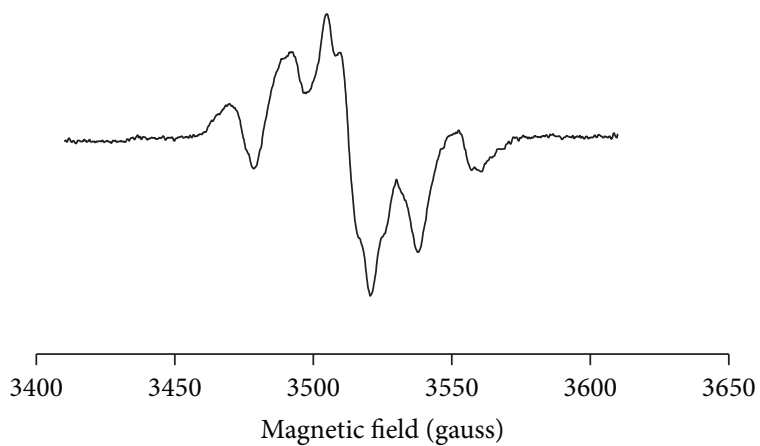

(b)

FIGURE 5: ESR spectra of $65 \mathrm{kGy}$ irradiated UHMWPE powder (pristine (a) and vitamin E blended (b)).

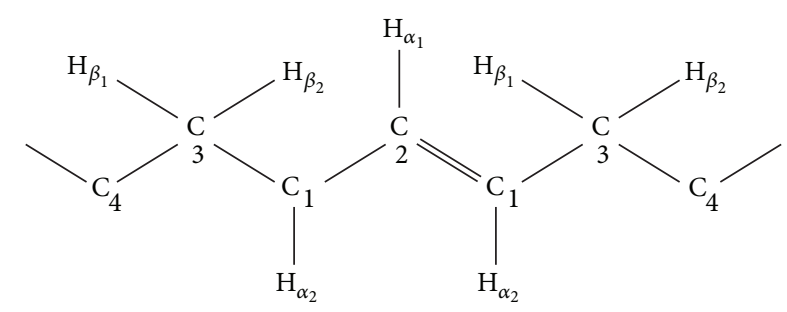

FIGURE 6: Configuration of "allyl type" radical showing the positions of $\alpha$ - and $\beta$-hydrogen atoms.

cross-link density [6]. Due to reduction in cross-link density the mechanical properties of polymer are also reduced which is not required at all for the case of orthopedic medical applications. However, for the detailed interpretation of the observed difference in radical decay and rate of radical decay further studies are in progress. 


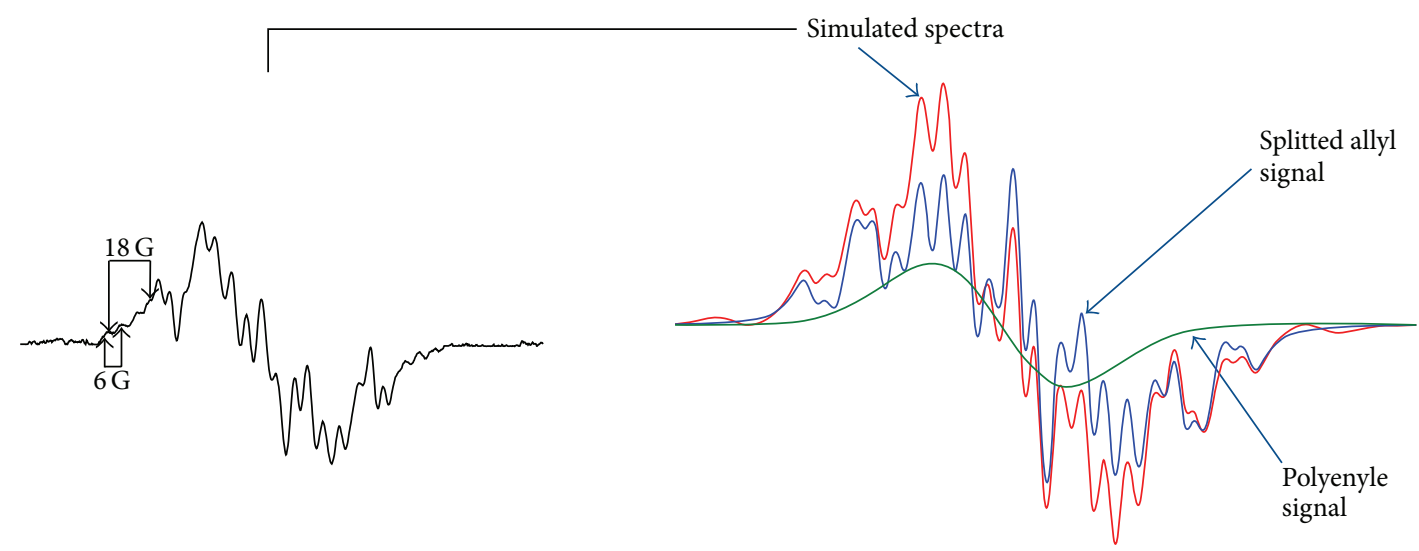

(a)

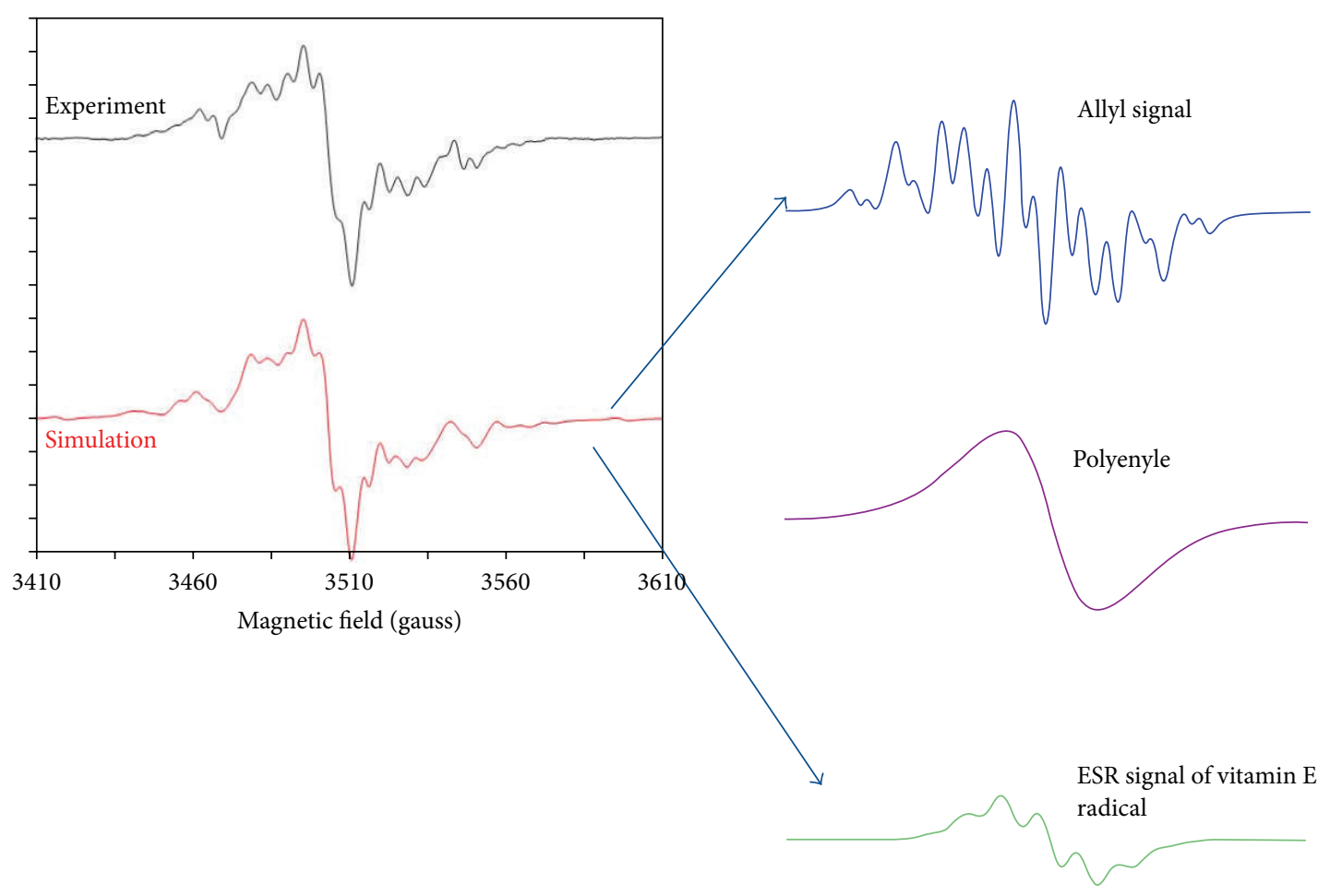

(b)

FIgURE 7: Spectrum deconvolution using WinSim software (a) pristine UHMWPE and (b) vitamin E blended UHMWPE.

\section{Conclusions}

The well-studied six-line pattern due to an alkyl radical and the seven-line pattern due to an allyl radical in the respective ESR spectrum of gamma-irradiated UHMWPE are produced by an average coupling/splitting from the nearby protons. Because the PE molecules are randomly oriented in the semicrystalline UHMWPE matrix, the protons (hydrogen atoms) at nearest ( $\alpha$-protons) and next nearest ( $\beta$-protons) positions from the unpaired electron site are indistinguishable. However, the production of super hyperfine (shf) lines ESR spectra for $100 \mathrm{kGy}$ in unoriented powder UHMWPE at room temperature, due to separate couplings of $\alpha$ - and $\beta$-protons with the paired electron at the free radical site, is reported for the first time, to our knowledge. The results further suggest that allyl radicals are $\sim 80 \%$ oriented. While similar spectra are produced by vitamin E-containing powder and consolidated solids, the super hyperfine pattern disappears in all samples in 24 hours in air. This hyperfine pattern in UHMWPE is missing for $65 \mathrm{kGy}$ irradiated samples under the same experimental conditions.

\section{Conflict of Interests}

The authors declare that there is no conflict of interests regarding the publication of this paper. 


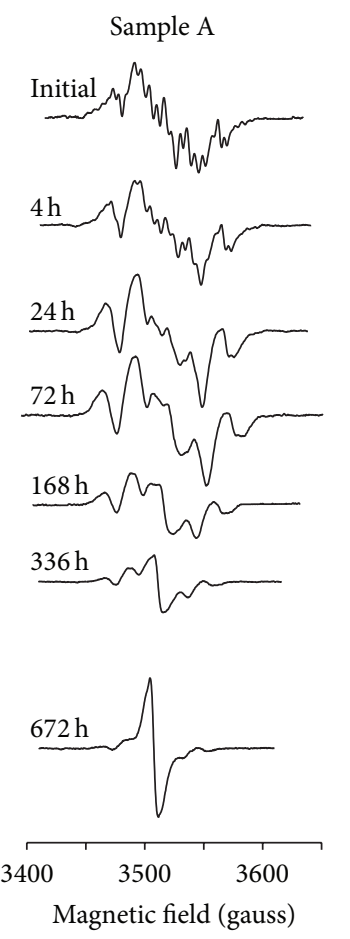

(a)

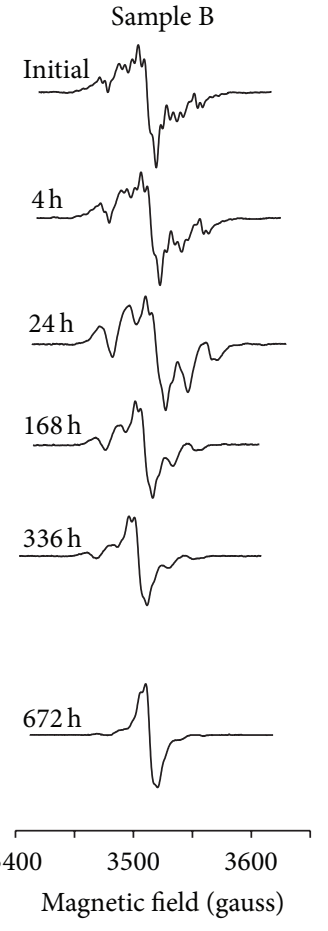

(b)

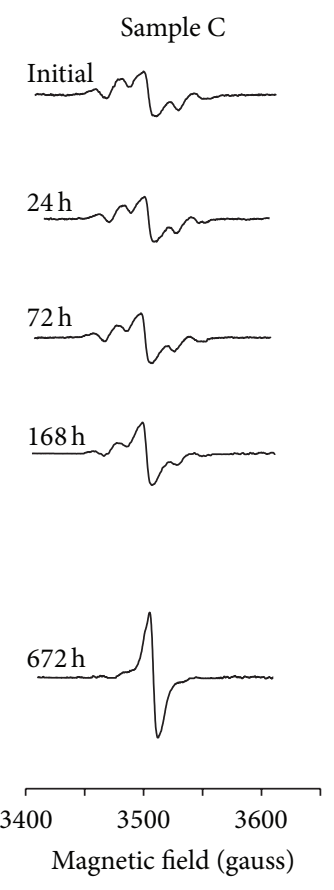

(c)
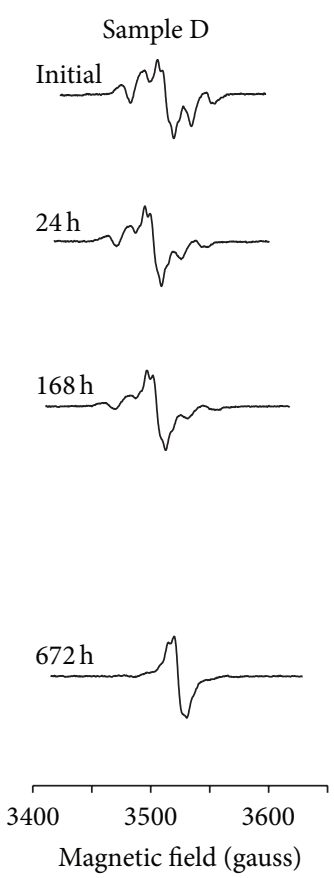

(d)

FIGURE 8: ESR spectrum plotted as a function of oxidation time in air for 678 hours: (Sample A) virgin UHMWPE powder 100-kGy irradiated; (Sample B) vitamin E blended UHMWPE powder 100-kGy irradiated; (Sample C) virgin UHMWPE powder 65-kGy irradiated; (Sample D) vitamin E blended UHMWPE powder 65-kGy irradiated. * (Note: all the spectra are on the same $y$-axis scale except Sample B. The scale of Sample B is twice the all other samples in the figure).

\section{Acknowledgments}

Dr. Malik Sajjad Mehmood would like to thank NSF Industry/University Cooperative Center for Biosurfaces (IUCB) at the University of Memphis, Ticona, USA, and UET Taxila, Pakistan, for their support during this research work. The moral support from his English teacher Ms. Tehmina Farrukh is also acknowledged here with core depth of his heart.

\section{References}

[1] S.-I. Ohnishi, Y. Ikeda, M. Kashiwagi, and I. Nitta, "Electron spin resonance studies of irradiated polymers I. Factors affecting the electron spin resonance spectra of irradiated polymers," Polymer, vol. 2, pp. 119-141, 1961.

[2] E. Oral, Y. Ikeda, and O. K. Muratoglu, "Radiation cross-linking in ultra-high molecular weight polyethylene for orthopaedic applications," Nuclear Instruments and Methods in Physics Research Section B: Beam Interactions with Materials and Atoms, vol. 265, no. 1, pp. 18-22, 2007.

[3] E. J. Lawton, R. S. Powell, and J. S. Balwit, "Effect of physical state during the electron irradiation of hydrocarbon polymers. Part II. Additional experiments and discussion pertaining to trapped radicals in hydrocarbon polymers," Journal of Polymer Science, vol. 32 , no. 125 , pp. $277-290$.

[4] P. O’Neill, C. Birkinshaw, J. J. Leahy, and R. Barklie, “The role of long lived free radicals in the ageing of irradiated ultra high molecular weight polyethylene," Polymer Degradation and Stability, vol. 63, no. 1, pp. 31-39, 1999.
[5] M. Shafiq, M. S. Mehmood, and T. Yasin, "On the structural and physicochemical properties of gamma irradiated UHMWPE/silane hybrid," Materials Chemistry and Physics, vol. 143, no. 1, pp. 425-433, 2013.

[6] M. S. Mehmood, B. M. Walters, T. Yasin et al., "Correlation of residual radical's with three phase morphology of UHMWPE: analysis for the dependence on heat involved during vitamin $\mathrm{E}$ diffusion," European Polymer Journal, vol. 53, no. 1, pp. 13-21, 2014.

[7] S.-I. Ohnishi, S.-I. Sugimoto, and I. Nitta, “Temperature dependence of the ESR spectrum of irradiated oriented polyethylene," The Journal of Chemical Physics, vol. 37, no. 6, pp. 1283-1288, 1962.

[8] Y. Zhao, M. Wang, Z. Tang, and G. Wu, "ESR study of free radicals in UHMW-PE fiber irradiated by gamma rays," Radiation Physics and Chemistry, vol. 79, no. 4, pp. 429-433, 2010.

[9] D. R. Duling, "Simulation of multiple isotropic spin-trap EPR spectra," Journal of Magnetic Resonance, Series B, vol. 104, no. 2, pp. 105-110, 1994.

[10] M. S. Jahan and J. Durant, "Investigation of the oxygen-induced radicals in ultra-high molecular weight polyethylene," Nuclear Instruments and Methods in Physics Research, Section B: Beam Interactions with Materials and Atoms, vol. 236, no. 1-4, pp. 166171, 2005.

[11] M. S. Mehmood, J. M. Shah, S. R. Mishra, and B. M. Walters, "The effect of high dose on residual radicals in open air irradiated $\alpha$-T UHMWPE resin powder," Radiation Physics and Chemistry, vol. 84, pp. 100-104, 2013. 
[12] M. D. Ridley and M. S. Jahan, "Effects of packaging environments on free radicals in $\gamma$-irradiated UHMWPE resin powder blend with vitamin E," Journal of Biomedical Materials Research. Part A, vol. 88, no. 4, pp. 1097-1103, 2009.

[13] M. S. Mehmood, T. Yasin, M. S. Jahan, B. M. Walters, M. Ahmad, and M. Ikram, "EPR study of $\gamma$-irradiated UHMWPE doped with vitamin E: assessment of thermal effects on the organic radicals during vitamin E diffusion," Applied Magnetic Resonance, vol. 44, no. 4, pp. 531-542, 2013.

[14] M. S. Mehmood, T. Yasin, M. S. Jahan et al., "Assessment of residual radicals in $\gamma$-sterilized shelf-aged UHMWPE stabilized with $\alpha$-tocopherol," Polymer Degradation and Stability, vol. 98, no. 6, pp. 1256-1263, 2013. 

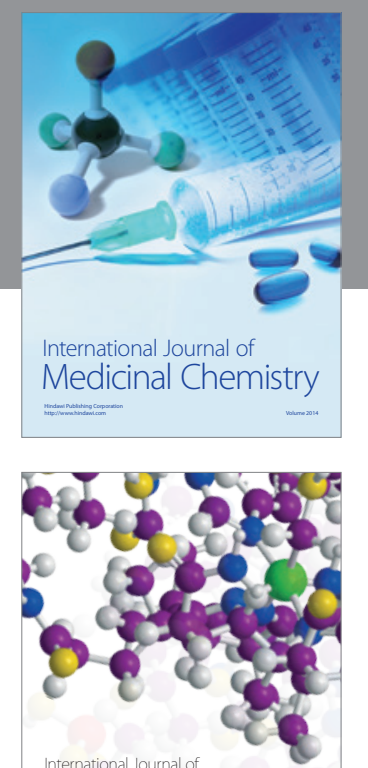

\section{Carbohydrate} Chemistry

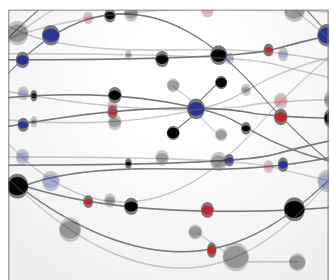

The Scientific World Journal
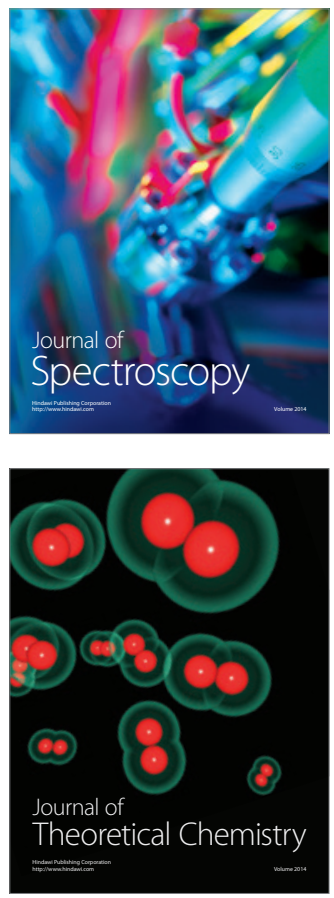
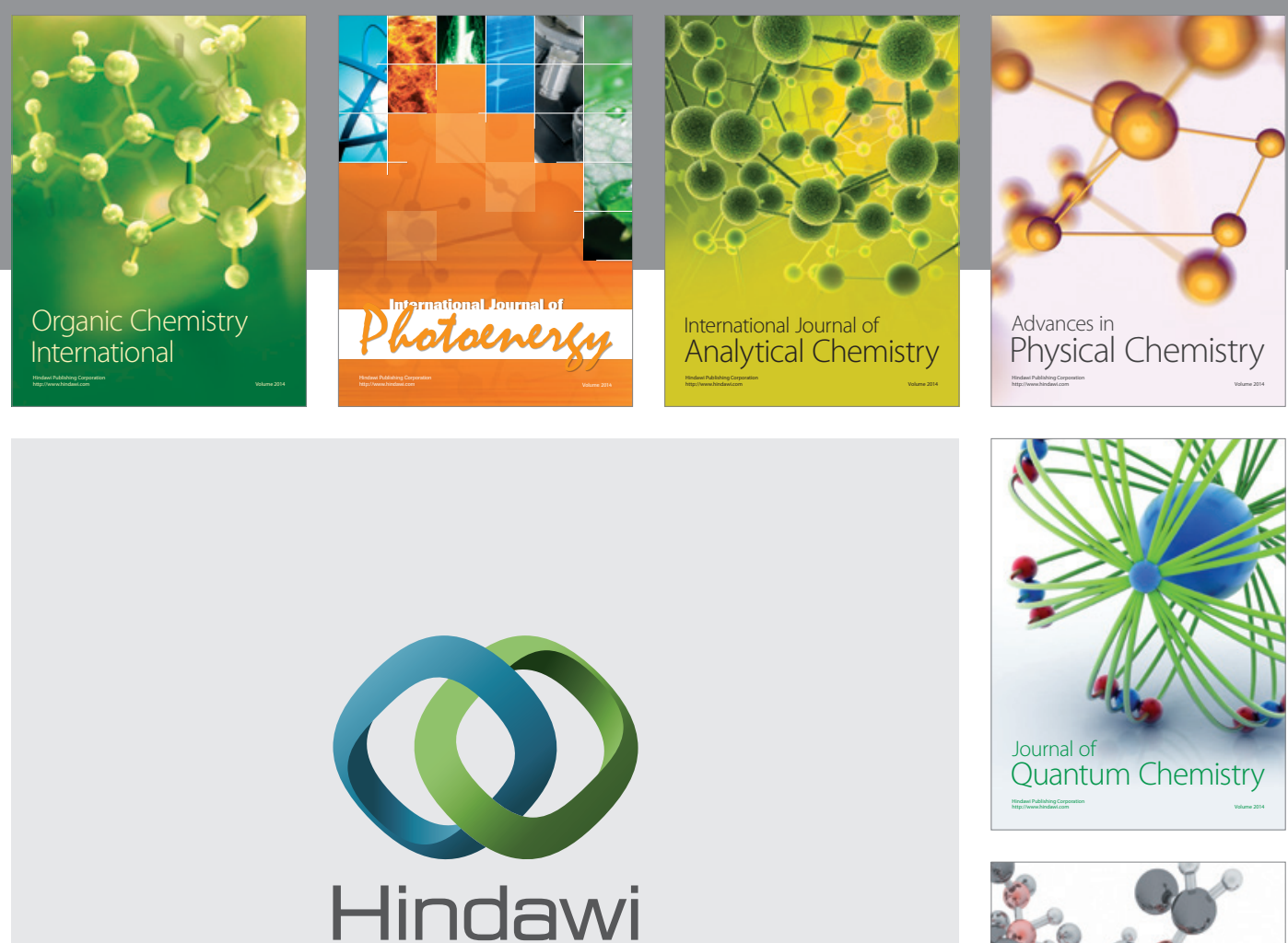

Submit your manuscripts at

http://www.hindawi.com

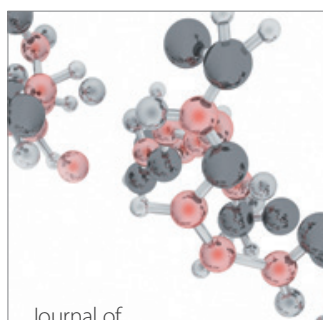

Analytical Methods

in Chemistry

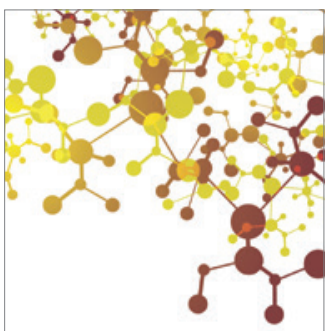

Journal of

Applied Chemistry

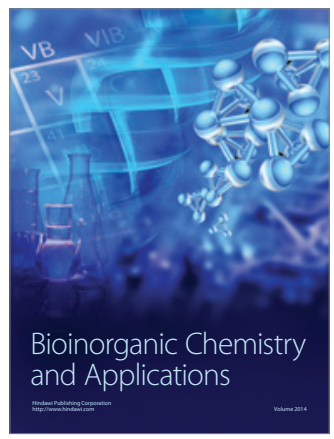

Inorganic Chemistry
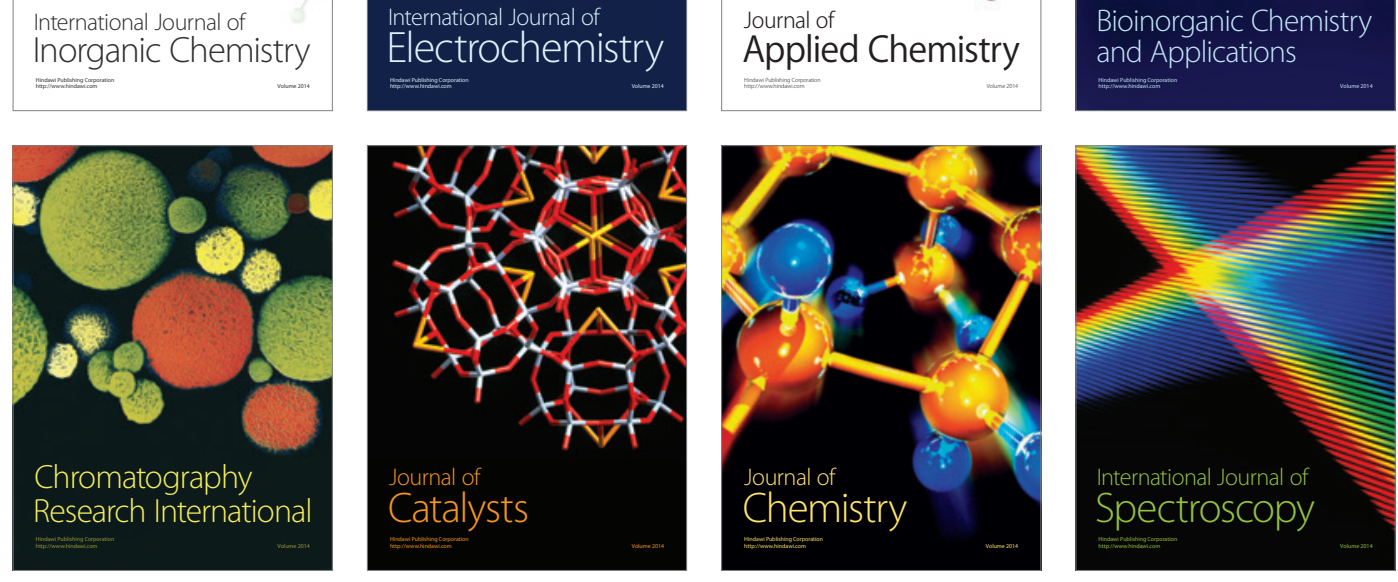